\author{
MARCO ARTURO MORENO CORRAL \\ INSTITUTO DE ASTRONOMÍA, UNAM
}

\title{
Ciencia y arte en dos publicaciones astronómicas novohispanas del siglo XVIII
}

U NO DE LOS GRANDES PROBLEMAS de la astronomía en el siglo XVIII era establecer con precisión la distancia entre la Tierra y el Sol, porque, entre otras cosas, ello permitiría determinar las dimensiones reales del sistema solar. Esa distancia, llamada Unidad Astronómica (UA), por su importancia científica, no podía derivarse de la teoría; debía obtenerse a partir de observaciones que permitieran medir un ángulo muy pequeño, conocido como paralaje solar. ${ }^{\mathrm{I}}$ En I716, Edmund Halley publicó un trabajo donde mos-

I. Se define como paralaje solar el ángulo desde el cual un observador hipotético localizado en el centro del Sol vería el radio terrestre. Al determinar su valor a partir de las observaciones puede encontrarse la magnitud absoluta (en kilómetros, millas, leguas o cualquier otra unidad de medida de longitud) de la distancia Tierra-Sol. Medir ese ángulo es difícil pues es muy pequeño, y por esto los errores (instrumentales y humanos) que se producen al hacerlo resultan del mismo orden, lo cual ocasiona alguna imprecisión inaceptable en su medida. Con el objeto de resolver este problema, Edmund Halley propuso que el tránsito fuera estudiado por el mayor número posible de observadores, distribuidos a lo largo y ancho de la extensa zona de la Tierra desde la cual sería visible ese fenómeno astronómico, y se midiera con gran exactitud la duración del paso de Venus por el disco solar, calculada en alrededor de seis horas. Particularmente se buscaba determinar los instantes en que el disco oscuro del planeta Venus, proyectado sobre la brillante superficie del Sol, tocaba, entraba completamente y salía de él. Debido a su diferente posición geográfica en la Tierra, cada observador vería a Venus proyectado en una posición distinta del disco solar, por lo que mediría un tiempo ligeramente diferente para cada uno de esos sucesos. Del análisis cuidadoso de esas diferencias y conociendo de forma precisa la ubicación de cada observador, podría determinarse el paralaje solar. 
tró que podría encontrarse el valor de la UA a partir de observaciones simultáneas, hechas por varios astrónomos, de un tránsito de Venus.

Pero ¿qué es un tránsito? En I627, Johannes Kepler mostró que esos sucesos astronómicos ocurrían debido al desplazamiento de Mercurio y Venus alrededor del Sol en órbitas elípticas, las cuales quedan contenidas dentro de la órbita de la Tierra, por lo cual en ocasiones es posible ver desde nuestro planeta a uno de ellos cruzarse sobre la brillante superficie solar, apareciendo proyectado sobre ella como un pequeño disco oscuro, que lentamente se desplaza y la atraviesa durante algunas horas. Kepler calculó que en I63 I ocurriría un tránsito de Venus, y Jeremias Horrocks, revisando sus datos, encontró que el siguiente tendría lugar en I639, el cual pudo observar desde su natal Inglaterra por algunos minutos.

Los siguientes tránsitos venusinos sucederían en 176I y en I769. Los astrónomos europeos se prepararon para estudiar el de 176I siguiendo el método ideado por Halley; pero aunque obtuvieron resultados alentadores, los datos no fueron concluyentes. Por esta razón se realizó un gran esfuerzo internacional para estudiar el de 1769 , el cual sería visible en un área grande del océano Pacífico, el sureste asiático y la parte oeste del hemisferio norte de América, esta última zona contenía el sur de la península de Baja California y una sección del centro de México. En la parte peninsular del territorio nacional se asentaron el novohispano Joaquín Velázquez de León ${ }^{2}$ y una comisión franco-española, quienes desde tres lugares localizados en la población de San José del Cabo realizaron con éxito las observaciones; ${ }^{3}$ mientras tanto, desde la ciudad de México José Antonio Alzate Ramírez, José Ignacio Bartolache y Antonio de León y Gama observaron el tránsito venusino.

\section{Observaciones desde la ciudad de México}

En las actas del ayuntamiento de la capital novohispana correspondientes a I769 puede leerse:

2. Santiago Ramírez, Ensayos biográficos de Joaquín Velázquez de León y Andrés Manuel del Río, México, Universidad Nacional Autónoma de México-Facultad de Ingeniería-Sociedad de Ex Alumnos, 1983, p. 78.

3. Jean-Baptiste Chappe d'Auteroche, Viaje a Baja California para la observación del tránsito de Venus sobre el disco del Sol, el 3 de junio de I760, Manuel Álvarez y Graciela Albert (trad. y notas), Marco Arturo Moreno Corral (introd.), Ensenada, Impresora del Noroeste, 2010. 
En el cabildo que celebró esta nobilísima ciudad en ocho de mayo de mil setecientos sesenta y nueve, el Sr. don Joseph Ángel de Cuevas y Aguirre dijo que siendo especie de la mayor utilidad para nuestros asuntos la observación del paso de Venus por el disco del Sol, tanto que los monarcas católico y cristianísimo han mandado sujetos de toda habilidad y satisfacción, venciendo las mayores distancias e incomodidades hasta llegar a California a celebrar la observación, le parecía muy propio del vínculo de la nobilísima ciudad, que contribuyendo a las ideas del soberano y haciendo este servicio a la República, encargase la misma operación a don Joseph Alzate y a don Joseph Bartolache, uno y otro, sujetos de inteligencia y empeño, como han manifestado, el primero en los diarios que dio al público, y el segundo con la sustitución de la cátedra de matemáticas, para que hasta donde alcance nuestro horizonte, formen su observación con el mayor cuidado, den cuenta de ella a este nobilísimo ayuntamiento y de su orden, la participen a los enviados para este asunto. ${ }^{4}$

También se les indicó dónde realizar las observaciones, pues en el mismo documento se apuntó: "porque la disposición de las casas capitulares puede ser la más acomodada y ventajosa para esta función, es a sí mismo de sentir que se les brindara, con sus azoteas para que en ellas formaran su observación y cálculo”. Aceptada esta comisión, Alzate y Bartolache se prepararon para realizar la observación del tránsito del 3 de junio de 1769 . Días antes, trasladaron a la parte alta del edificio señalado los telescopios y cronómetros que utilizarían y los orientaron adecuadamente mediante el trazo cuidadoso de la línea meridiana que pasaba por su punto de observación. 5 De esa manera regularon el paso de los relojes de acuerdo con sus observaciones astronómicas, lo que les permitió determinar con precisión las coordenadas geográficas de su observatorio. Estos procedimientos son sencillos de resumir, pero si se recuerda que julio es un mes de lluvias abundantes en la capital mexicana y las operaciones debían realizarse con exactitud y tiempo, podrá valorarse adecuadamente el mérito de aquellos observadores capitalinos.

Sobre la forma como procederían el día de la observación, Bartolache comunicó a los regidores lo siguiente:

4. Archivo Histórico del Distrito Federal "Carlos de Sigüenza y Góngora", Actas de Cabildo, Historia, "Nombramiento de los Sres. Bartolache y Alzate para ir a hacer las observaciones del paso de Venus por el disco del Sol", I769, exp. Io.

5. La línea meridiana o del mediodía es la línea determinada por la intersección de los planos del meridiano y del horizonte del observador. Sirve sobre todo para fijar con precisión la dirección norte-sur. 
El 3 de junio examinaremos (con el favor de Dios) desde media mañana empleando todo el día en este trabajo, que nos será glorioso, cediendo en honor y siendo por encargo de la ciudad imperial México, que va a dar en esta ocasión una prueba visible de que su policía ${ }^{6}$ no es inferior a la de las ciudades más cultas y que tiene educados en su seno y formados de suyo sin maestro ni escuela, algunos ciudadanos que gustan de la astronomía. Yo destinaré de entre mis cursantes aquellos que me parecieren más hábiles, ${ }^{7}$ para lo que allí se viere hacer, conforme a la instrucción que a todos daré por escrito. En fin daremos cuenta a su excelencia de la resulta y a los caballeros matemáticos que pasaron a la California, según se me previene. ${ }^{8}$

Llegada la fecha, la observación se realizó de manera exitosa y así lo comunicaron Alzate y Bartolache al cabildo:

llenos del más profundo respeto y veneración a la grandeza de vuestra excelencia le presentamos hoy la resulta de aquel trabajo, que no puede menos que sernos glorioso habiéndolo tomado, no sólo bajo los auspicios y alta protección de vuestra excelencia, sino también por su orden positivo, que nos comunicaron en carta misiva los dos señores comisionados.

Sí, señor excelentísimo, el 3 de junio presente ha ofrecido a México un doble fenómeno: Venus pasando por el disco solar, y una multitud de jóvenes americanos a la faz del público observando el pasaje, con aparatos y prevenciones de un observatorio formal. Se puede dudar cuál de esos dos fenómenos tuvo mayor número de observadores, el astronómico o el político. Lo cierto es que este último era más raro, que aquel que en I 639 a 24 de noviembre, en I76 I en 6 de junio 9 y, ahora últimamente aconteció en los cielos el mismo aspecto [...] Una tercera vez en fin nos ha proporcionado el cielo la bella ocasión de comenzar nosotros alguna: y no debe parecer muy tarde para dar principio a este género de ocupaciones, que apenas, aun apenas se podían esperar en nuestra República. ${ }^{\text {Io }}$

6. Bartolache usó aquí la palabra policía con un sentido amplio de organización y reglamentación interna de una sociedad, no solamente como el de un cuerpo encargado de velar por el bien público.

7. En esas fechas, Bartolache sustituía a Velázquez de León en la cátedra de Astronomía y Matemáticas de la Real y Pontificia Universidad de México.

8. Actas de Cabildo, doc. cit., ff. 6 y 7.

9. Aluden a los tránsitos venusinos de 1639 y I76r.

Io. Actas de Cabildo, doc. cit., f. I7. 
El 2I de julio de I769, en el cabildo de la capital novohispana se leyó el informe presentado por Alzate y Bartolache. Los regidores Ángel de Cuevas Aguirre y Avendaño y Joseph Matheos se expresaron sobre el particular diciendo:

somos testigos oculares del trabajo, que expendieron en ellos, como también del que tuvieron en adquirir los instrumentos physicos, y en hacer construir a sus expensas algunos que no se pudieron hallar. El día tres de junio estaba ya todo dispuesto y las azoteas de las casas de ayuntamiento eregidas en un verdadero observatorio: finalmente hicieron la observación, y si hemos de juzgar su éxito por la habilidad de los observadores, por las prudentes precauciones que tomaron y por el escrupuloso esmero con que la hicieron, puede desde luego vuestra excelencia asegurarse de la felicidad de la empresa. ${ }^{\text {II }}$

Finalmente, con relación al aspecto administrativo de este asunto, Juan Lucas de Lassaga, destacado personaje en el terreno técnico de la minería novohispana, como miembro de aquel cabildo escribió:

Que la relación que presentan en 6 f. útiles se imprima sin dilación alguna a expensas de vuestra excelencia dando previamente cuenta de ello al excelentísimo seńor virrey marqués de Croix e impetrando su venia; supuesto lo cual, se dedique y salga bajo los auspicios del soberano nombre del rey. Para que así conste haberse interesado esta muy noble y muy leal ciudad motu proprio en este asunto, que de hecho ha estimado útil y recomendable nuestro católico monarca. ${ }^{12}$

\section{Publicación sobre el tránsito venusino}

En 1769, Bartolache y Alzate publicaron en la ciudad de México una hoja suelta titulada Suplemento a la famosa observación del tránsito de Venus por el disco del Sol (fig. I). ${ }^{13}$ Indicaron lo siguiente: "Los autores presentaron à su tiempo la relación de esta i otras observaciones curiosas, que ia se publicarán. Entre tanto i para satisfacción de todos, dan este cortísimo suplemento que toca à la

I I. Ibidem, f. 25.

I2. Ibidem, f. 26.

13. El título completo de ese reporte fue Suplemento a la famosa observación del tránsito de Venus por el disco del Sol, hecha de encargo de la Mui Noble Imperial México por D. Jph. Ig. ${ }^{\circ}$ Bartolache, i D. Jph. Ant. ${ }^{\circ}$ Alzate el 3 de Junio de 1769. 


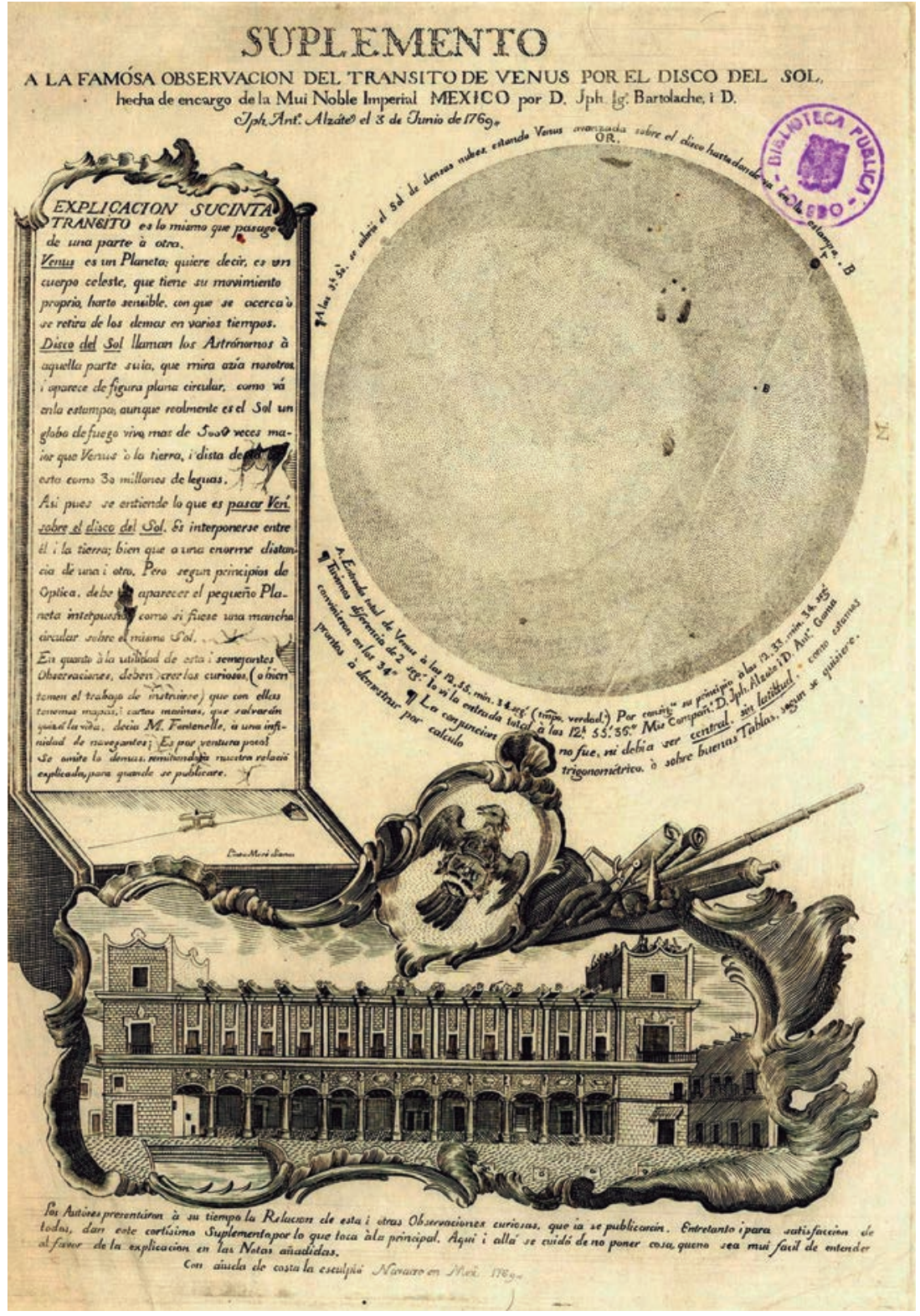

I. El tránsito de Venus de 1769, observado desde la ciudad de México por José Ignacio Bartolache y José Antonio Alzate Ramírez. Imagen digital cortesía de la Biblioteca Pública de Toledo, España. La trayectoria que siguió Venus se señala con la cuerda dibujada del lado derecho del disco solar. La letra A indica por donde entró el planeta y la B hasta dónde lo vieron nuestros personajes antes de que el Sol se ocultara en el horizonte. 
principal". A pesar de los esfuerzos para localizar la relación en seis fojas citada en el párrafo anterior, no se le ha hallado en los acervos mexicanos de libros y manuscritos antiguos, ${ }^{\mathrm{I} 4}$ lo cual hace pensar que nunca se publicó y solamente salió a luz la hoja mencionada. En apoyo de esta idea, se consultó la obra de Medina La imprenta en México. ${ }^{15}$ En el tomo VI, en la parte correspondiente a los ańos I769-I770, no se reporta ninguna obra de Bartolache o de Alzate con ese nombre o tema, pero en la página 93 del mismo volumen, refiriéndose Medina a este último y a sus Gacetas de Literatura de México publicadas en I772, escribió: "núms. 9 y Io de I4 y I6 de diciembre con un Suplemento y lámina (falta)" y puso la nota: "Se imprimió en Puebla I83I $4^{\circ}$. En las pp. I37I 48 del tomo IV de esta reimpresión, se podrá ver el contenido de los ns. que faltan y la lámina del suplemento".

Consultado ese tomo de la edición poblana de $\mathrm{I} 83 \mathrm{I},{ }^{\mathrm{I}}{ }^{6}$ vimos que en efecto aparece dicha lámina, la cual se muestra aquí como figura 2, pero para sorpresa nuestra es algo diferente a la publicación original.

La rareza de ese grabado novohispano del siglo XVIII (fig. I) y su valor como obra científica y artística de aquella época, aunados al reciente tránsito venusino del 5 de junio de 2012 , observado desde gran parte del territorio nacional por muchos mexicanos interesados en ese suceso —el cual sólo volverá a ocurrir el II de diciembre de 2II7-,${ }^{17}$ nos convenció de la necesidad de rescatar tan notable y bello documento colonial mexicano.

Aunque el texto es fácil de leer, a continuación presentamos su transcripción; en ella se buscó mantener la distribución, la forma y el tamaño, y solamente se omitieron las ilustraciones.

I4. Por ejemplo, se ha buscado en el Archivo Histórico del Distrito Federal, en la Biblioteca Nacional de México, la Biblioteca del Congreso, la Biblioteca México, las bibliotecas Lafragua y Palafoxiana del estado de Puebla, la Biblioteca Pública de Guadalajara y la Biblioteca Francisco de Burgoa de Oaxaca.

I5. José Toribio Medina, La imprenta en México (I539-I82I), t. VI (I768-I794), edición facsimilar, México, Universidad Nacional Autónoma de México, I989.

I6. Gacetas de Literatura de México: por D. José Antonio Alzate Ramirez, socio correspondiente de la Real Academia de las ciencias de París, del Real Jardin Botánico de Madrid y de la Sociedad Bascongada. Reimpresa en la oficina del hospital de S. Pedro, a cargo del ciudadano Manuel Buen Abad, Puebla, i83i.

I7. En esa fecha no será visible desde nuestro país, pero sí podrá observarse el que ocurrirá ocho ańos después, el 8 de diciembre de 2125. 


\section{SUPLEMENTO \\ A LA FAMÓSA OBSERVACION DEL TRANSITO DE VENUS POR EL DIS- CO DEL SOL,} hecha de encargo de la Mui Noble Imperial MEXICO por D. Jph Ig. ${ }^{\circ}$ Bartolache, i D Jph. Ant. ${ }^{\circ}$ Alzáte el 3 de Junio de 1769

\section{EXPLICACION SUCINTA}

TRANSITO es lo mismo que pasage de una parte à otra

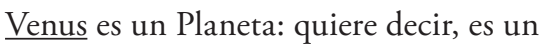
cuerpo celeste, que tiene su movimiento proprio, harto sensible, con que se acerca ò se retira de los demas en varios tiempos. Disco del Sol llaman los Astrónomos à aquella parte suia, que mira azía nosotros, i aparece de figura plana circular, como vá en la estampa; aunque realmente es el Sol un globo de fuego vivo, mas de 5000 veces maior que Venus ò la tierra, i dista de esta como 30 millones de leguas.

Asi pues se entiende lo que es pasar $\underline{V e n} .{ }^{s}$ de

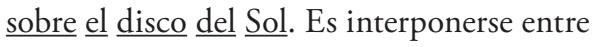
él i la tierra; bien que a una enorme distancia de una i otro. Pero según principios de Optica, debe aparecer el pequeño Planeta interpuesto como si fuese una mancha circular sobre el mismo Sol.

En quanto à la utilidad de esta i semejantes Observaciones, deben crer los curiosos, (o bien tomen el trabajo de instruirse) que con ellas tenemos mapas, i cartas marinas, que salvarán quizá la vida, decía M. Fontenelle, à una infinidad de navegantes. ¿Es por ventura poco? Se omite lo demás, remitiéndo à nuestra relación explicada, para quando se publicare. 
IA las $3 .^{\text {h }} 50$ '. se cubrió el Sol de densas nubes, estando Venus avanzada sobre el disco hasta donde va en la estampa. B

A. Entrada total de Venus à las I2. 55. min. 3.4. seg. ${ }^{\mathrm{s}}\left(\mathrm{t}_{\mathrm{mp}} \mathrm{O}\right.$. verdad:) Por consig. ${ }^{\text {te }}$ su principio a las I2. 33. min. 34. seg. ${ }^{5} /$ Tuvimos diferencia de 2 seg. ${ }^{5}$ Io vi la entrada total à las I2. ${ }^{\text {h }} 55^{\prime} \cdot 36^{\prime}$. Mis Compañ..$^{\mathrm{s}} \mathrm{D}$. Jph. Alzate i D. Ant. ${ }^{\circ}$ Gama/convinieron en los $34^{\prime \prime}$. La conjuncion no fue, ni debia ser central, sin latittud, como estamos/prontos à demostrar por calculo trigonométrico ò sobre buenas Tablas, segun se quisiere.

Los Autóres presentáron à su tiempo la Relacion de esta i otras Observaciones curiosas, que ia se publicarán. Entretanto i para satisfaccion de/todos, dan este cortísimo Suplemento por lo que toca àla principal. Aqui i allá se cuidó de no poner cosa, queno sea mui facil de entender/al favor dela explicacion de las Notas añadidas.

Con aiuda de costa la esculpió Navarro en Mex. ${ }^{\circ} 1769$

La figura I es una excelente reproducción obtenida de la imagen digital de alta resolución que la Biblioteca Pública de Toledo, España, nos proporcionó para utilizarla en otras investigaciones. ${ }^{18}$ Según la información de la institución toledana, el original es una hoja de grabado calcográfico en folio. Como puede apreciarse, se encuentra dividida en cuatro partes. En el encabezado, los autores indican lo que tratan y por orden de quién lo hicieron. Al lado izquierdo se encuentra un recuadro donde se explica detalladamente qué es un tránsito de Venus. En el lado derecho aparece representado el disco solar, tal como lo observaron los autores en aquella fecha, pues, además de indicar la trayectoria seguida por Venus, incluyeron las manchas solares presentes el 3 de junio y un curioso aspecto granuloso que, como se comentará adelante, pudo tratarse de una de las manifestaciones de la actividad solar. Alrededor del Sol, con una distribución circular, pusieron los datos astronómicos obtenidos durante la observación. Finalmente, en la parte baja, un dibujo representa las casas de cabildo enmarcadas por una orla, donde al centro de la misma están el

I8. José Manuel Vaquero et al., "Two Early Sunspots Observers: Teodoro de Almeida and José Antonio Alzate”, Solar Physics, núm. 240, 200I, pp. 165-175; José Manuel Vaquero y Marco Arturo Moreno Corral, "Historical Sunspot Records from Mexico", Geofísica Internacional, vol. 3, núm. 47, 2008, pp. I89-192. 
escudo de la ciudad de México y algunos elementos representativos del trabajo científico hecho por Alzate y Bartolache.

Al final del grabado puede leerse claramente: "Con aiuda de costa la esculpió Navarro en Mex. I769", comprobándose así que el grabador de este curioso documento científico-artístico fue José Mariano Navarro (I742-ca. I809), quien por aquella fecha tuvo su taller en la calle de Donceles de la capital novohispana. De ese artesano interesado en aspectos artísticos ${ }^{19}$ se conocen varios trabajos que ilustraron diversas publicaciones novohispanas en aquellos años. ${ }^{20}$

Al comparar directamente las figuras i y 2 se advierte con claridad que la segunda, la cual ya no aparece firmada, es una copia pobre de la primera. Las diferencias principales entre las dos hojas se encuentran en el grabado de las casas de cabildo, que en la versión original tiene gran detalle, pues, como puede verse en la ampliación mostrada en la figura 3, el frente de esa construcción tiene mayor número de arcos en la planta baja y más ventanas en el primer piso. En la copia de I83I, a éstas se les quitaron los balcones; además, la amplia escalera que va directamente al exterior, en el segundo grabado ya no tiene detalle.

También hay diferencia en el texto que circunda al Sol. En la copia publicada en las Gacetas, el disco solar es de menores dimensiones y la explicación a su alrededor más apretada, lo que dificulta la lectura.

Finalmente, la figura 5, que amplía la parte central de las casas de cabildo y la torre oriente de las mismas, muestra con claridad a los observadores del tránsito. Se puede apreciar entre el centro de ese edificio y aquella torre la presencia de un par de observadores, uno de los cuales asiste al otro, que mira a través del telescopio. Sobre el torreón, un grupo nutrido observa por al menos cinco telescopios, mientras que al otro lado de la calle ${ }^{2 \mathrm{I}}$ puede verse a otro observador solitario apuntando con su telescopio hacia el cielo. Todos estos detalles han desaparecido en la copia publicada en Puebla.

La parte ampliada del grabado original reproducido en la figura 4 muestra el escudo de armas otorgado a la ciudad de México por Carlos

19. Efraín Morales Castro, "Un grabador neoclásico", Anales del Instituto de Investigaciones Estéticas, vol. 9, núm. 33, 1964, pp. I07-III.

20. Por ejemplo, produjo y firmó los grabados de los mapas de la Nueva Espańa y de Baja California, así como el del Templo Mayor que aparecen en la Historia de la Nueva España, escrita por su esclarecido conquistador Hernán Cortés, aumentada con otros documentos y notas, por el ilustrísimo señor don Francisco Antonio Lorenzana, arzobispo de México, México, Imprenta del Superior Gobierno del Br. D. Joseph Antonio de Hogal en la Calle de Tiburcio, I770.

2I. Lo que actualmente corresponde al edificio ubicado donde, a partir del Zócalo, comienza la calle 5 de Febrero. 


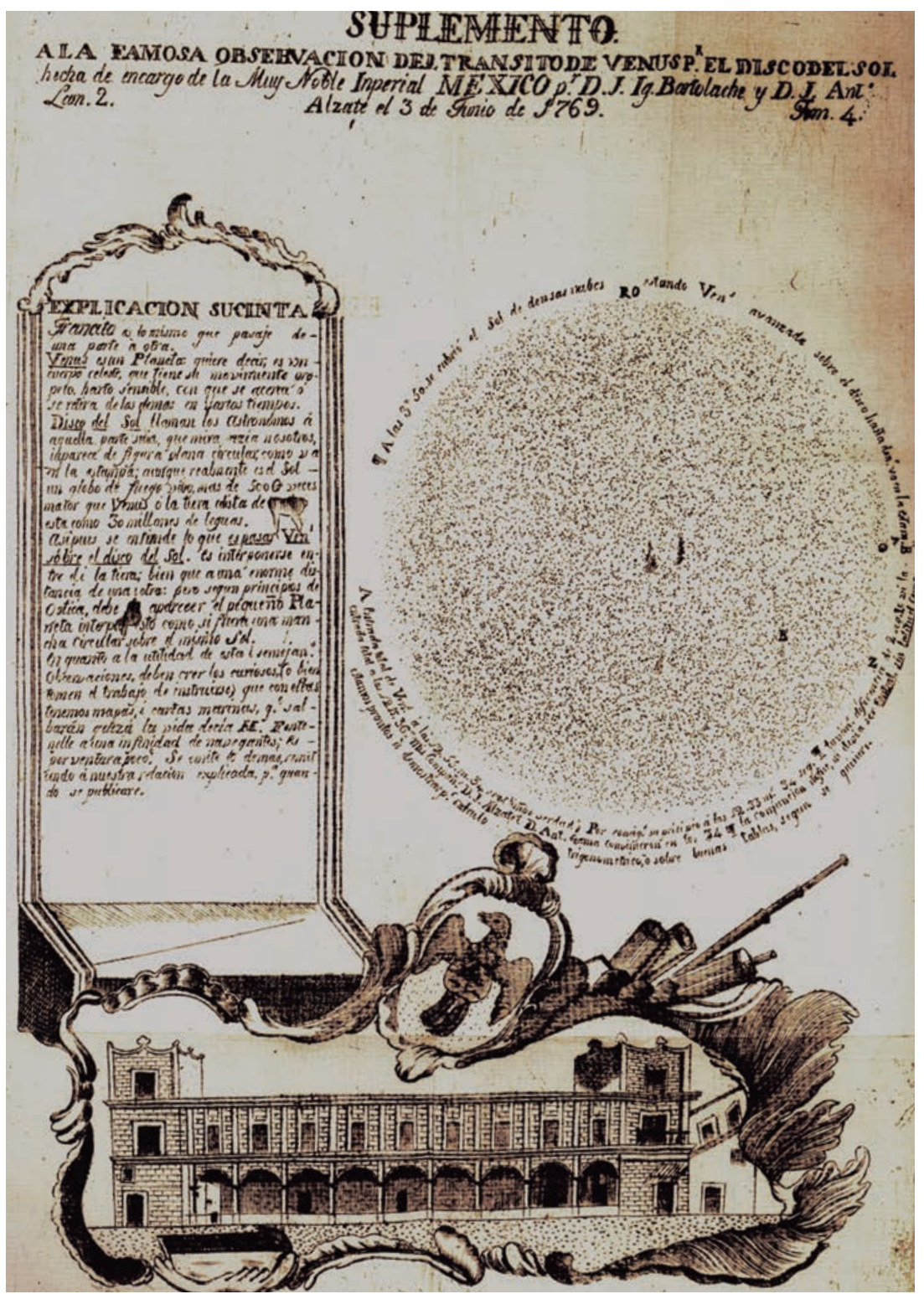

2. Lámina sobre el tránsito venusino de 1769 en las Gacetas de Literatura de México, publicadas en Puebla en I83I. 


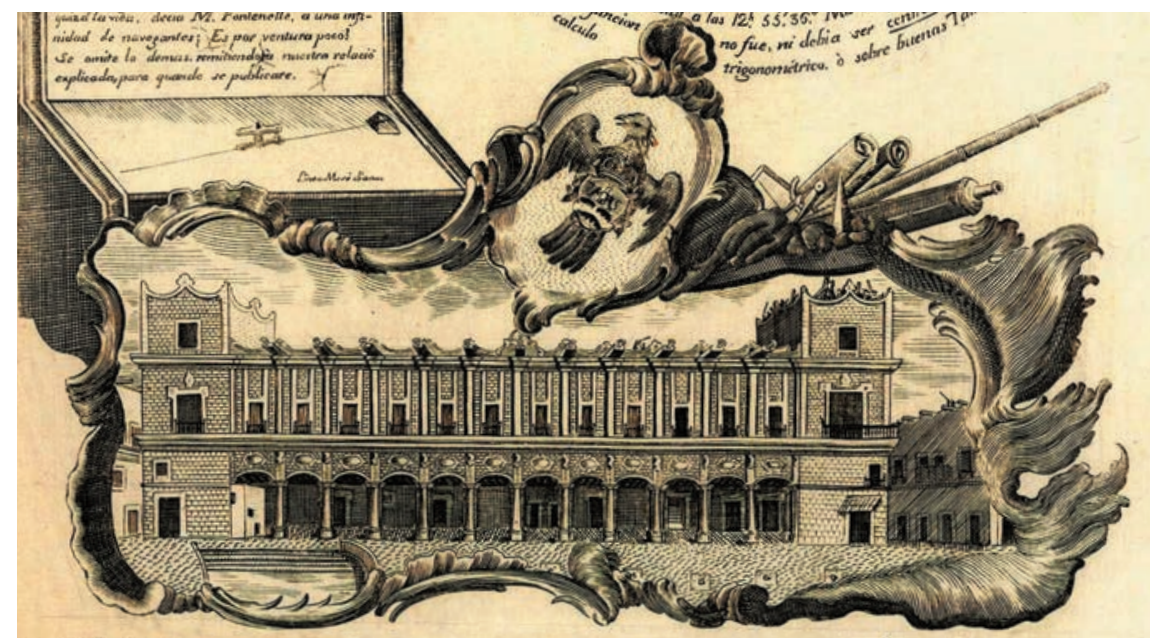

3. Detalle de la parte inferior de la figura I, donde se muestran las casas de cabildo, el escudo de la ciudad de México, los instrumentos astronómicos y la línea meridiana del recuadro.

$\mathrm{V},{ }^{22}$ mientras que en la copia publicada en las Gacetas se han perdido los detalles de los leones rampantes cobijados por la corona.

El original también muestra en la parte baja del recuadro la frase "Línea meridiana” y cómo se trazó, información que ya no aparece en la copia. Por último, respecto del grabado original, es apropiado comentar que sobre la orla en torno a las casas de cabildo aparecen —en clara alegoría al tema tratado, pues se dibujaron símbolos entonces asociados con el quehacer astronómicoel compás, la parte posterior de dos telescopios, parte de un cuadrante, ${ }^{23}$ una figura geométrica, así como un par de documentos enrollados.

\section{El edificio del ayuntamiento de la ciudad de México en 1769}

La parte del grabado que representa las casas de cabildo merece un comentario más amplio, sobre todo por tratarse de una imagen detallada de dicha edifica-

22. Este escudo puede verse en la pared del edificio antiguo del Gobierno del Distrito Federal. Fue elaborado en mosaico y, junto con otros escudos, adorna el portal de esa sede gubernamental comprendido entre la avenida 20 de Noviembre y la calle 5 de Febrero.

23. Instrumento astronómico pretelescópico que recibe ese nombre por ser la cuarta parte de un círculo. Este aparato era muy utilizado por los observadores para determinar la latitud del sitio. 
4. Sección amplificada del grabado de Navarro, que claramente muestra sobrepuesto el escudo que Carlos V otorgó a la ciudad de México.

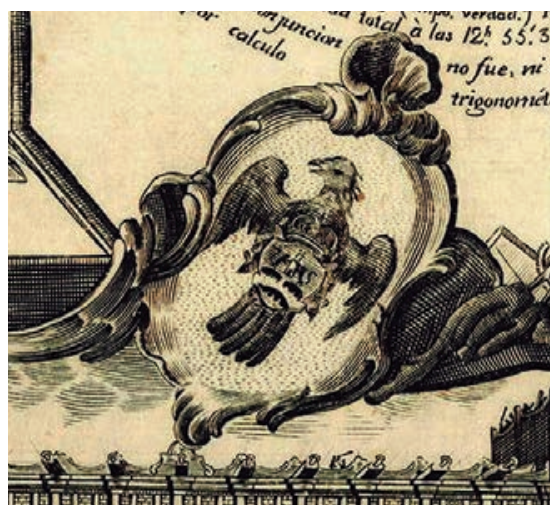

ción y poco conocida en la actualidad. Las representaciones existentes de ese edificio se inician hacia I596, con un dibujo esquemático que muestra la Plaza Mayor de la ciudad de México. Ese trabajo anónimo se ha reproducido en diversas publicaciones ${ }^{24}$ donde hemos podido consultarlo y ver que sitúa esa edificación en su posición actual en el Zócalo capitalino, identificándola en forma manuscrita con la leyenda "delantera de las casas del ayuntamiento". Un poco después, en I628, Juan Gómez de Trasmonte produjo el plano que muestra una vista de la ciudad de México. En él, la "casa de cabildo" se identifica con la letra C. El original de ese plano, titulado "Forma y levantado de la ciudad de México", actualmente se halla en la Biblioteca Medicea Laurenziana de Florencia, Italia. Hay una copia moderna en la colección del Instituto de Investigaciones Estéticas de la Universidad Nacional Autónoma de México. ${ }^{25}$ Aunque en esos documentos se identifica el ayuntamiento de la ciudad de México, realmente su representación tiene poco detalle, de modo que casi nada puede saberse a partir de ellos sobre la estructura original de esa histórica edificación.

Al finalizar el siglo Xvir hubo un motín de la población indígena de la capital novohispana. Carlos de Sigüenza y Góngora narró los hechos ocurridos el 8 de junio de $1692,{ }^{26}$ indicando que a consecuencia de aquellos distur-

24. Véase Fernando Benítez, Historia de la ciudad de México, México, Salvat, 1984, t. 2, pp. 26-27.

25. Roberto L. Meyer, “Trasmonte y Boot. Sus vistas de tres ciudades mexicanas del siglo XVII", Anales del Instituto de Investigaciones Estéticas, vol. XXVII, núm. 87, otońo de 2005 , pp. I77-I98.

26. Carlos de Sigüenza y Góngora, Alboroto y motín de los indios de México, México, Univer- 


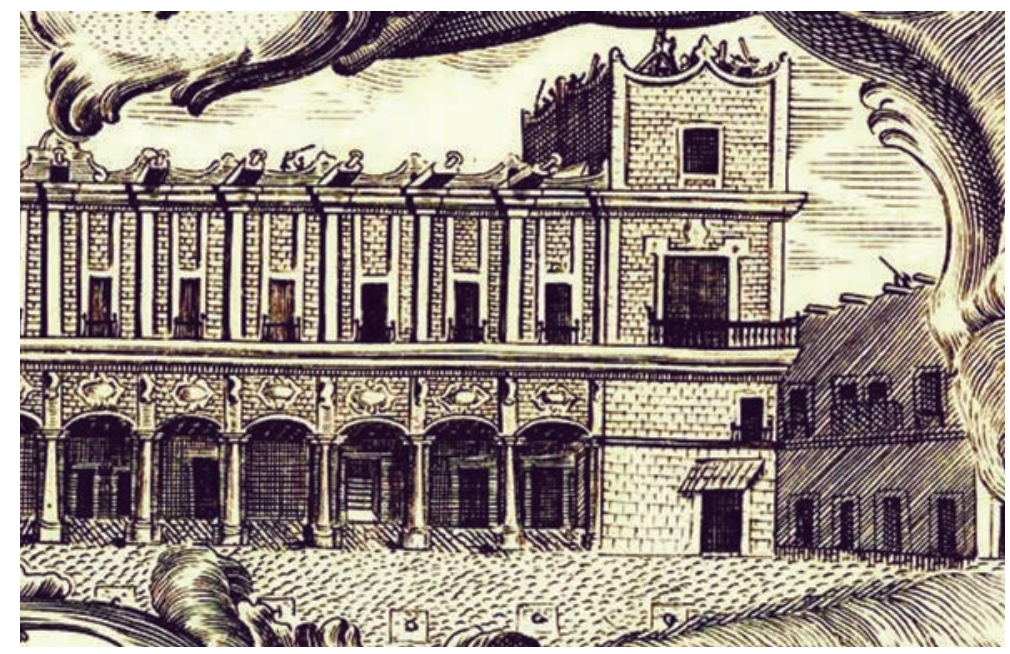

5. Parte central-oriente de las casas de cabildo de la ciudad de México, donde claramente se puede ver a los observadores y sus telescopios.

bios resultó dañado seriamente el edificio del ayuntamiento. En la pintura de la Plaza Mayor de Cristóbal de Villalpando de $1695^{27}$ quedaron registrados los daños que sufrió el palacio virreinal en ese motín. Lamentablemente, la perspectiva que el pintor dio a las casas de cabildo no permite decir mucho, aunque parecieran estar en proceso de reconstrucción. En cambio, sí pueden verse el pasillo techado tan característico de esa edificación y la acequia real que pasa exactamente por el frente, corriendo en dirección este-oeste, la cual en aquella época llevaba agua y permitía el desplazamiento de chalupas y trajineras. A la altura del ayuntamiento, en la pintura de Villalpando, había tres puentes permanentes sobre dicha acequia que, por la escala de los individuos que los cruzan, debieron tener al menos unos cinco metros de largo. A decir de Vetancurt, en su Teatro mexicano de 1695, "por las calles donde hay acequias

sidad Nacional Autónoma de Méxco-Coordinación de Humanidades/Miguel Ángel Porrúa (Biblioteca Mexicana de Escritores Políticos), I986. Véase también Josefina Muriel, "Una nueva versión del motín del 8 de junio de I692", Estudios de Historia Novohispana, núm. I8, I998, pp. I07-II5.

27. Benítez, op.cit., pp. 66-7I. 
6. El tránsito de

Mercurio observado desde la ciudad de México, el 9 de noviembre de 1769 . Imagen digital cortesía de la Biblioteca Pública de Toledo, España. La trayectoria seguida por Mercurio se seńala con la cuerda dibujada del lado izquierdo. Los discos oscuros sobre el disco del Sol son las manchas solares presentes el día de la observación.

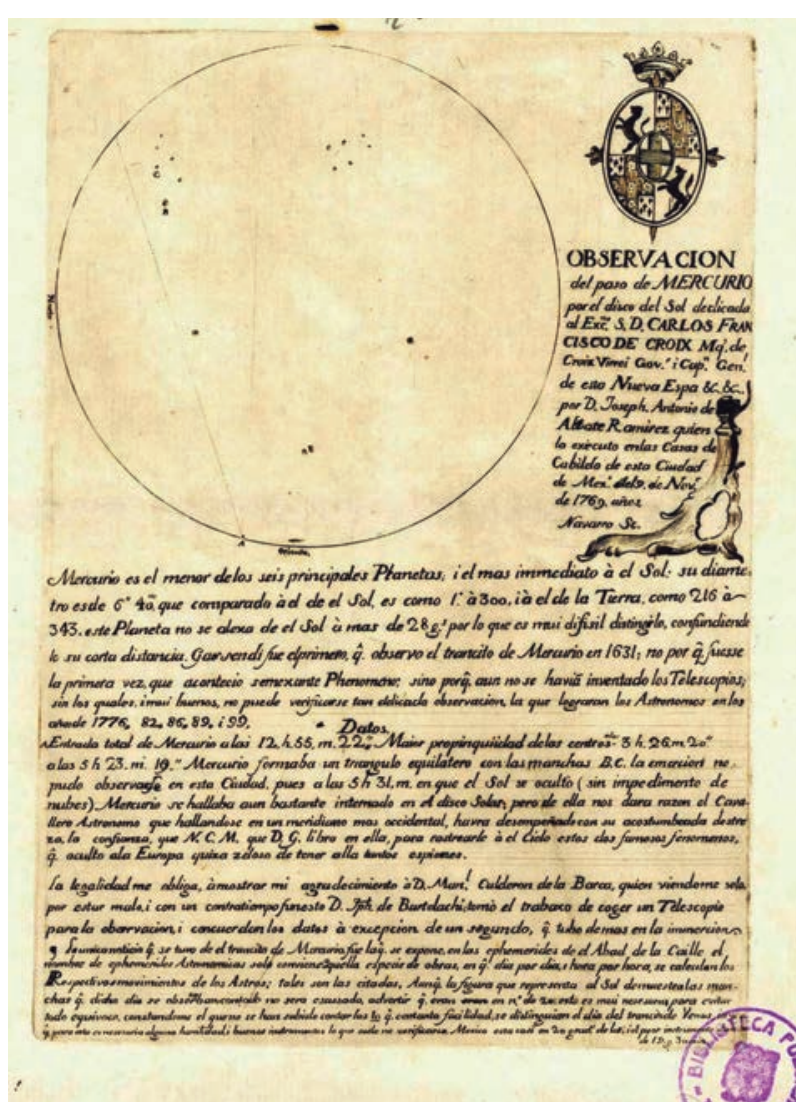

tienen puentes de calicanto fuertes”. ${ }^{28}$ En la representación de Villalpando aún no se aprecian las características torres que identifican el ayuntamiento.

En los planos de Pedro de Arrieta de 1737 y de Carlos López del Troncoso de 1760 , las casas de cabildo aparecen representadas; sin embargo, estos documentos tampoco brindan detalles precisos sobre tan importante edificación capitalina. Hasta donde hemos podido investigar, el grabado de 1769 del edificio del ayuntamiento — realizado por Navarro e incluido en el reporte de las observaciones de Alzate y Bartolache sobre el tránsito venusino que aquí nos

28. Apud Roberto Moreno, "La ciudad de México", en Historia de México, México, Salvat Mexicana, I978, vol. 6, p. I419. 
ocupan- es la primera representación detallada de ese histórico edificio y por ello debe ser conocida, sobre todo por los especialistas, para que se hagan las valoraciones pertinentes.

Seguramente Alzate y Bartolache la incluyeron para indicar el lugar desde donde realizaron las observaciones y como reconocimiento al mandato que recibieron de las autoridades capitalinas. Como ya se ha dicho, esa parte del documento tiene en la zona central superior la representación de un águila de frente con las alas abiertas parcialmente, sobre la cual se dibujó de manera simplificada el escudo que Carlos V le otorgó a la ciudad de México el 4 de julio de $1523 .{ }^{29}$ En la figura 4, sobre el pecho del águila se mira la torre que representa a México-Tenochtitlan, flanqueada por los dos leones rampantes que clavan sus garras sobre ella, simbolizando la toma y posesión de la capital azteca por los españoles, lo cual se indica además por la presencia de la corona. Aunque el escudo ilustrado en el documento, grabado por Navarro, no se adoptó oficialmente para la ciudad de México en 1534, sin duda cumplía con la intención de señalar con claridad que aquellas observaciones se hicieron en la capital de la Nueva Espańa. Aquí es pertinente indicar que durante el siglo XVIII otros documentos llevaron representaciones similares, como por ejemplo el escudo situado en el margen superior derecho del Plano de la ciudad de México dividida en cuarteles de Manuel Villavicencio, publicado en I782.

Volviendo al dibujo de las casas de cabildo, puede apreciarse que Navarro lo elaboró desde una perspectiva central y mirando perpendicularmente a su fachada, la cual muestra con detalle los característicos I 2 arcos del portal de la planta baja subsistentes hasta el presente, así como los I2 balcones de la planta superior y los dos de los torreones oriente y poniente. En este grabado hecho mirando de norte a sur, el lado derecho corresponde al oeste y el izquierdo al este.

En la parte frontal del ayuntamiento, en el grabado de Navarro, puede verse la acequia real — todavía en funciones en la pintura de Villalpandoya parcialmente tapada, pues solamente se mira un segmento en el lado este de las casas de cabildo adonde, según esa ilustración, se puede llegar desde el ayuntamiento por una amplia escalinata de cinco peldaños, que aparentemente son restos de un embarcadero. El flujo del agua y la dirección del barandal del puente indican que la acequia seguía el mismo curso observado en el cuadro de 1695 .

29. Antonio de León Pinelo, Recopilación de Indias, Ismael Sánchez Bella (ed. y est. prel.), México, Miguel Ángel Porrúa, I992, t. III, p. 2039. 
Sobre el empedrado frente al edificio se miran cinco estructuras rectangulares espaciadas regularmente; bien podrían ser obstáculos colocados intencionalmente para impedir el tráfico de vehículos o, quizá, soportes para amarrar bestias, pues tienen algo semejante a argollas. Estas suposiciones son razonables, sobre todo si se toma en cuenta la actividad representada en la pintura de I695, aunque en realidad no se cuenta con datos sobre el particular.

Toda la información comentada en este apartado, valiosa para los estudiosos del desarrollo urbano de la ciudad de México, es un elemento más para justificar el rescate y la publicación del grabado de Navarro.

\title{
El tránsito de Mercurio
}

El 9 de noviembre del mismo año (I769) ocurrió otro tránsito, pero en esa ocasión el planeta que cruzó frente al disco solar fue Mercurio. Alzate observó también ese suceso astronómico y volvió a publicar los resultados en una hoja suelta, ilustrada en la figura 6.

Navarro también grabó esta hoja, como puede leerse al final de la dedicatoria que nuestro astrónomo hizo al virrey Carlos Francisco de Croix, donde escribió "Navarro Sc". Como en el caso anterior, para comodidad del lector se presenta la transcripción del documento.

\author{
OBSERVACION \\ del paso de MERCURIO \\ por el disco del Sol dedicada \\ al Exc. ${ }^{\text {mo }}$. S.D. CARLOS FRAN \\ CISCO DE CROIX Mq $\mathrm{Me}^{\mathrm{s}}$ de \\ Croix Virrei Gov. Cap ${ }^{\mathrm{n}}$ Gen ${ }^{1}$. \\ de esta Nueva Espa\&.\&., \\ por D. Joseph Antonio de \\ Alzate Ramirez quien \\ la executo en las Casas de \\ Cabildo de esta Ciudad \\ de Mex.o el 9 de Nov. ${ }^{e}$ \\ de I769. años \\ Navarro Sc.
}


Mercurio es el menor de los seis principales Planetas; i el mas inmediato à el Sol: su diame-/tro es de 6' 4 " que comparado à el de el Sol es como I à 3 oo i el de la Tierra como 216 à 343 , este Planeta no se alexa de el Sol à mas de $28 \mathrm{~g}^{\mathrm{s}}$ por lo que es mui difisil distingirlo, confundiendo/lo su corta distancia. Gassendi fue el primero $\hat{\text { q }}$ observo el trancito de Mercurio en I63I; no por $\hat{\mathrm{q}}$. fuesse/la primera vez que acontecio semexante Phenomeno; sino porâ. aun no se havià inventado los Telescopios; sin los quales, i mui buenos, no puede verificarse tan delicada observacion la que lograron los Astronomos en los/años de I776. 82. 86. 89. i 99.

Datos

Entrada total de Mercurio a las I2.h. 55.m. 22”. Maior propinquiidad de los centros alas 3 h. $26 \mathrm{~m}$. 20 "/alas 5 h 23.m I9." Mercurio formaba un triangulo equilatero con las manchas B. C. la emercion no/pudo observarse en esta Ciudad, pues a las 5h 3 I.m. en que el Sol se oculto (sin impedimento de/nubes) Mercurio se hallaba aun bastante internado en el disco Solar: pero de ello nos dara razon el Cava-/llero Astronomo que hallandose en un meridiano mas occidental, havra desempeñado con su acostumbrada destre-/za, la confianza, que N.C.M. que D.G. libro

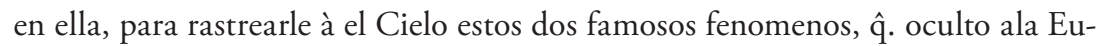
ropa quizá zeloso de tener alla tantos espiones.

La legalidad me obliga àmostrar mi agradecimiento à D. Man ${ }^{1}$ Calderon de la Barca, quien viendome solo/por estar malo i con un contratiempo funesto D. Jph de Bartolache; tomò el trabaxo de coger un Telescopio/para la obcservación, i concuerdan los datos à excepción de un segundo, $\hat{\mathrm{q}}$. tubo demas en la inmercion. ILa unica noticia $\hat{q}$. se tuvo del trancito de Mercurio fue la $\hat{q}$. se expone en la ephemerides de el Abad de la Caille el/nombre de ephemerides Astronomicas solo conviene a aquella especie de obras, en $\hat{\mathrm{q}}$ dia por dia, i hora por hora, se calculan los/Respectivos movimientos de los Astros; tales son las citadas. Aunq̂ la figura que representa al Sol demuestra las man-/chas $\hat{\text { q }}$. dicho dia se observaban, con todo no será escusado advertir $\hat{q}$. eran en n. ${ }^{\circ}$ de 20 : esto es mui nesesario para evitar: todo equivoco constandome el que no se han sabido contar las $\underline{\text { lo }} \hat{\mathrm{q}}$. con tanta facilidad, se distinguían el dia del trancito de Venus i $\hat{\text { q }}$. para esto es necesaria alguna havilidad, i buenos instrumentos, lo que suele no verificarse. Mexico esta

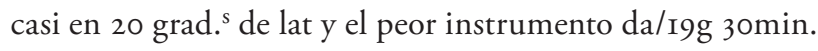

La figura 6 es también una excelente digitalización proporcionada por la Biblioteca Pública de Toledo, España, pues hasta el momento no ha sido posible 
ubicar ese grabado en ninguna de nuestras bibliotecas antiguas. Según esa institución, se trata de "I h. de grab.; $4^{\circ}$. La hoja es un grabado calc.".

En ella, Alzate representó el disco solar en el lado superior izquierdo del grabado. El oriente aparece indicado en la parte inferior del Sol y el norte a la izquierda. La trayectoria seguida por Mercurio fue marcada por una línea punteada. Como en el grabado antes comentado, también indicó las manchas solares presentes el 9 de noviembre, y en el texto aclaró que en total eran 20. La posición marcada con A fue la parte donde se dio el primer contacto geométrico entre los discos de los dos cuerpos involucrados. A lo largo del texto describió lo observado y midió e informó que en esa ocasión no tuvo la ayuda de Bartolache, quien estaba enfermo. Lo asistió en la observación D. Man[ue]l Calderón de la Barca, personaje que no ha podido ser identificado.

El grabado del tránsito de Mercurio es sencillo comparado con el del tránsito venusino, ya que, además de la figura del disco solar, solamente presenta el blasón del virrey Carlos Francisco de Croix. Éste es un documento menos conocido que el primero, razón por la cual lo hemos considerado en este trabajo.

\section{Comentarios}

Los grabados que se han presentado aquí son prácticamente desconocidos en la actualidad y, como ya se ha mencionado, no ha sido posible localizarlos en ninguno de los acervos de documentos antiguos de nuestro país. Probablemente ello obedezca a que fueron publicados como hojas sueltas y luego, una vez pasada la curiosidad causada por los sucesos astronómicos que reportan, se desecharon. Los únicos ejemplares que con toda certeza existen se encuentran en el extranjero, lo cual seguramente se debe a que Alzate, como socio correspondiente de la Academia de Ciencias de París, del Real Jardín Botánico de Madrid y de la Sociedad Bascongada, los hizo llegar a miembros de esas agrupaciones académicas, tal como lo ha mostrado en un artículo muy completo la investigadora del Centre de Recherche en Histoire des Sciences et des Techniques, Patrice Bret, ${ }^{30}$ para el caso francés.

30. Patrice Bret, "Alzate y Ramírez et l’Académie Royale des Sciences de Paris: La Réception des travaux d'un savant du Nouveau Monde", en Patricia Aceves Pastrana (ed.), Periodismo cientifico en el siglo XVIII: José Antonio Alzate y Ramírez, México, Universidad Autónoma 
Por los grabados conocidos de José Mariano Navarro, puede afirmarse que preferentemente realizó trabajos para ilustrar documentos técnicos y científicos. Ése fue el caso de los mapas de la Nueva España y de la península de Baja California usados en la edición de Lorenzana de las Cartas de relación de Hernán Cortés ya citadas. También a ese grabador se debe la ilustración del opúsculo de Alzate sobre el eclipse lunar que observó a finales de 1769 , donde representó los principales accidentes de la superficie de nuestro satélite, así como los mecanismos causantes de ese tipo de eclipses. ${ }^{3 \mathrm{I}}$ Igualmente se debe a Navarro la ilustración aparecida en las Lecciones de matemáticas de Ignacio Bartolache, grabado de gran complejidad que muestra, en alegoría a la antigua Grecia, las aplicaciones de la geometría. ${ }^{32}$

Volviendo a los dos grabados de los tránsitos planetarios, varios comentarios surgen de su estudio. Primeramente debe mencionarse que ambos fueron concebidos como documentos científicos donde se consignaron los datos relevantes de aquellas observaciones. A pesar de esa característica, no fueron escritos en un lenguaje complicado, ni mucho menos en latín, que era la lengua franca de la ciencia en aquella época. Alzate, además de dejar constancia de los parámetros de importancia astronómica deducidos de la observación, buscó transmitir a un público amplio dicha información, haciéndolo en forma sencilla y en un lenguaje apropiado. Para garantizar el éxito de este proceso, resultaron de gran importancia los cuidadosos grabados realizados por Navarro. Este hecho ha permitido aprovecharlos incluso en la actualidad, pues, gracias al cuidado de ese artista novohispano al representar lo que vieron nuestros astrónomos, esos documentos han servido recientemente para publicar artículos científicos sobre las manchas solares y la granulosidad de la superficie solar. ${ }^{33}$ Seguramente esa fidelidad en la reproducción de los detalles de la superficie del Sol fue producto de la meticulosidad con que Navarro realizaba sus obras, pero también se debió a las magníficas observaciones realizadas por nuestros astrónomos y a la calidad de sus instrumentos.

Metropolitana (Estudios de Historia Social de las Ciencias Químicas y Biológicas, 6), 200I. 3I. José Antonio Alzate Ramírez, Eclypse de luna del doce de diciembre de mil setecientos sesenta y nueve años: observado en la imperial ciudad de México, México, impreso por Joseph Jáuregui, I770.

32. Lecciones de matemáticas que en la universidad de México dictaba D. Josef Ignacio Bartolache, México, Imprenta de la Biblioteca Mexicana, 1769.

33. José Vaquero, "An Early Drawing of Solar Granulation?", Revista Mexicana de Física, núm. 57, 2011, pp. I56-I57. 
Respecto del grabado del tránsito de Venus, que como ya se mencionó es el más elaborado, la representación de las casas de cabildo localizada en la parte inferior, hasta donde se ha podido investigar, no se ha publicado posteriormente, lo cual hace de este documento una fuente más para el estudio de los edificios coloniales, en particular del inmueble que ocupa actualmente la jefatura de gobierno del Distrito Federal en el Zócalo de la ciudad de México.

Tocante al segundo grabado, el menos conocido, se sabe que ya en el siglo XIX eran muy pocos quienes lo habían visto. Francisco Jiménez, uno de los astrónomos mexicanos más importantes de la segunda mitad de esa centuria, mencionó en un artículo sobre los tránsitos planetarios ${ }^{34}$ que la plancha metálica utilizada para hacerlo ya había sido destruida. En el trabajo citado reprodujo en una lámina el grabado de Navarro, que muchos años después se volvió a publicar. ${ }^{35}$ s

34. Francisco Jiménez, "Pasos de Mercurio y Venus por el disco del Sol, observados en México y en California en I769", Boletín de la Sociedad Mexicana de Geografía y Estadística, $2^{\mathrm{a}}$ época, vol. 4, 1872, pp. 94-105.

35. Marco Arturo Moreno Corral, "Los tránsitos de Mercurio", Anuario del Observatorio Astronómico Nacional, año CVI, I985, pp. I73-175.

N.B. Quiero agradecer a la Biblioteca Pública de Toledo, España, por haberme proporcionado sin restricciones las imágenes digitales de las dos publicaciones hechas por José Antonio Alzate Ramírez, conservadas en su Fondo Antiguo con los números de identificación 4-23182(39) y 4-23I80(40). Las gestiones para obtenerlas las realizó José M. Vaquero del Departamento de Física, Universidad de Extremadura, Mérida, España, a quien le agradezco haberme proporcionado las copias utilizadas en este trabajo. También quiero reconocer el trabajo de apoyo que María Elena Jiménez, responsable de la Biblioteca del Instituto de Astronomía de la Universidad Nacional Autónoma de México, Campus Ensenada, me ha brindado, al conseguir documentos antiguos relacionados con ésta y otras investigaciones realizadas sobre la historia de las ciencias exactas en el México colonial. Finalmente quiero agradecer los comentarios de dos árbitros anónimos, que sin duda ayudaron a mejorar este trabajo.

* Artículo recibido el 27 de julio de 20I2; aceptado el I6 de octubre de 2012. 